\title{
Subsurface Particle Analysis using X-ray Computed Tomography and Confocal X- ray Fluorescence
}

\author{
Nikolaus L. Cordes ${ }^{1}$, Srivatsan Seshadri ${ }^{2}$, George J. Havrilla ${ }^{1}$, Brian M. Patterson ${ }^{1}$, Michael Feser ${ }^{2}$, \\ Xiaoli Yuan ${ }^{3}$, Ying $\mathrm{Gu}^{3}$ and Deming Wang ${ }^{3}$ \\ ${ }^{1 .}$ Los Alamos National Laboratory, Los Alamos, NM, USA \\ 2. Carl Zeiss X-Ray Microscopy, Inc., Pleasanton, CA, USA \\ 3. Julius Kruttschnitt Mineral Research Center, University of Queensland, Indooroopilly, Brisbane, \\ Australia
}

Rapid screening of particulate samples is critical in confirming or denying the presence of elements of interest. Typically, high atomic number particles found on waste air filters or precious metals within minerals require a complex dissolution and/or chemical analysis to detect them, without giving any information on size or shape morphology. For this measurement, electron microscopy (which is performed separately) is required. However, secondary electron microscopy-based energy dispersive spectroscopy is limited to the top $1 \mu \mathrm{m}$ to $5 \mu \mathrm{m}$ of the sample; using this technique to interrogate elements below the sample surface typically involves destructive methods, such as focused ion beam (FIB) ablation. Micro X-ray fluorescence spectroscopy (micro-XRF) instruments, while able to detect sub-surface particles, are limited in that they cannot easily distinguish surface from sub-surface particles. The depth discrimination limitations of micro-XRF can be overcome by confocal micro-XRF [1] which allows for the spatial discrimination of X-ray fluorescence signals in all three spatial axes and allows for the detection, identification, and (with standards and modeling) quantification of the elements present at depth. Data is acquired by rastering the sample, acquiring one voxel at a time; hence, large samples can require days or even weeks to fully acquire a 3D elemental image. This analysis time can be significantly reduced by pre-screening the samples for X-ray absorption values of interest by utilizing a complementary, non-destructive X-ray imaging technique: micro X-ray computed tomography (microCT). This technique rapidly collects absorption contrast images of a sample and reasonable 3D data sets can be acquired within a few hours. While discrete elemental information is difficult with micro-CT, particles composed of high atomic number elements can be differentiated from particles composed of low atomic number elements based on X-ray attenuation. Using the particle location information, the prescreened particles can be targeted individually for in situ elemental analysis which rapidly decreases the time required to locate and elementally identify particles of interest. In this case study, a cylindrical mineral sample (with a diameter of $1.67 \mathrm{~mm}$ and height of $1.79 \mathrm{~mm}$ ) was prepared by mixing and compressing mineral particles $(\sim 10 \mu \mathrm{m}$ to $200 \mu \mathrm{m}$ in size) composed of $\mathrm{Cu}, \mathrm{Pb}, \mathrm{Pt}, \mathrm{Ni}, \mathrm{Fe}$ and trace elements. The samples were then imaged non-destructively with an Xradia MicroXCT-200 micro-CT scanner at $90 \mathrm{kV}, 8 \mathrm{~W}$ using a $10 \mathrm{X}$ objective with an isotropic voxel size of $\sim 1.85 \mathrm{um}$ in about 2 hours. Two high-Z particles of interest ( $\sim 20 \mu \mathrm{m}$ in diameter) were located (one surface particle and one subsurface particle) and targeted for confocal MXRF analysis. The locations of the particles are indicated in Figure 1A. Particle A is located at the polished surface of the mineralogical sample (Fig. 1B). Particle B (Fig. 1A) is located $\sim 300 \mu \mathrm{m}$ below the sample surface. Gold-plated tungsten wires were placed on the surface of the mineralogical sample, indicating the location of Particle A and the lateral position of Particle B, to aid in identifying spatial regions of interest using the confocal micro-XRF instrument, utilizing a Ag X-ray tube source operated at $45 \mathrm{kV}, 20 \mathrm{~W}$. Using these fiducial markers, the confocal micro-XRF beam was brought to these locations and an elemental spectrum of each particle was collected in $10 \mathrm{~s}$ for Particle A and $900 \mathrm{~s}$ for Particle B. Prior to this study, Particle A was identified as a 
tetraferro-platinum particle by mineral liberation analysis which is confirmed by the confocal microXRF. Particle B shows significant X-ray fluorescence peaks for both platinum and lead (Fig. 2). Due to the sampling volume of the confocal MXRF instrument ( 30 $\mu \mathrm{m}$ x $30 \mu \mathrm{m}$ x $65 \mu \mathrm{m})$, significant spectral contamination with adjoining particles is present. An improved instrument with higher spatial resolution optics would reduce this spectral contamination. These results show that coupling these two X-ray based imaging techniques allows for the spatial location, size measurement and elemental identification of high-Z particles within a matrix, in a non-destructive manner and without chemical/physical dissolution at depth.

\section{References:}

[1] Patterson, B. M.; Campbell, J.; Havrilla, G. J, X-Ray Spectrometry, 39 (2010), p. 184.

[2] This work was partially funded by National Science Foundation, under the award number NSF IIP1248744.
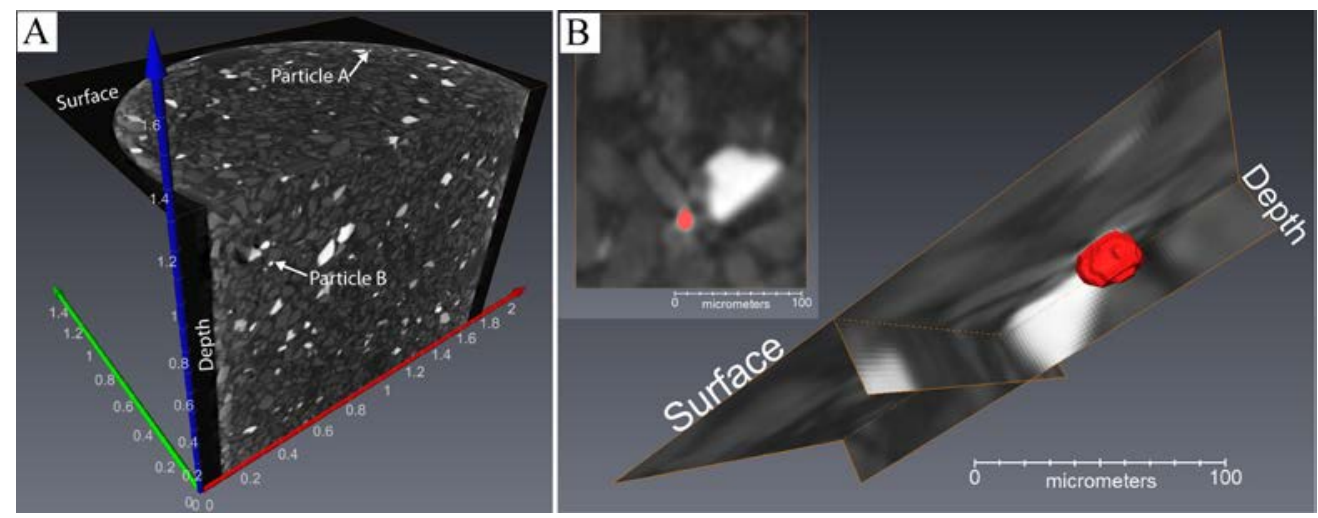

Figure 1. (A) Micro X-ray computed tomographic reconstructed slices of a mineralogical sample, highlighting the surface location of Particle A and subsurface location of Particle B. Scale is in millimeters. (B) Volume rendering of Particle A (particle shown in red). Micro X-ray computed tomographic reconstructed XY (surface) slice, YZ (depth) slice, and XZ (depth) slice are shown, highlighting the volume of Particle $A$ that is located beneath the surface of the mineral sample matrix. Insert: Reconstructed XY (surface) slice of Particle A (particle shown in red).

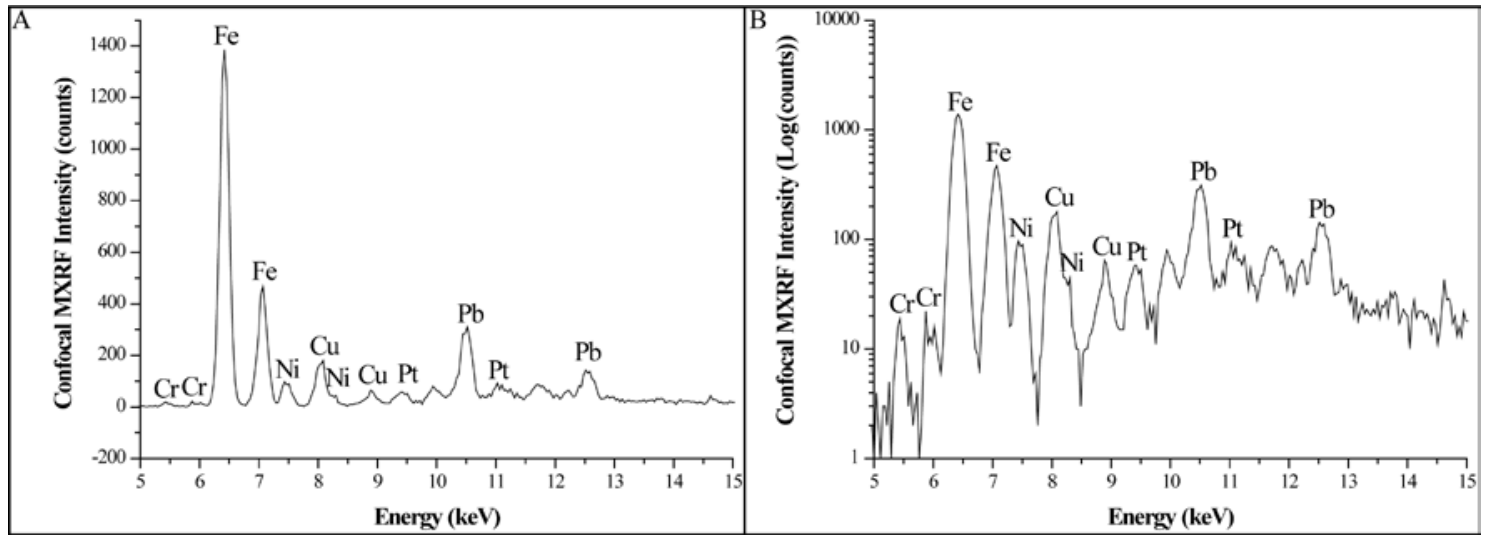

Figure 2. (A) Confocal micro X-ray fluorescence spectrum of Particle B, acquired from $~ 300 \mu \mathrm{m}$ below the sample surface. Spectrum was acquired with a 900 second dwell time. Spectral contamination is observed due to the small size of the particle compared to the instrument probe volume. (B) Spectrum of Particle B using a Log scale on the $\mathrm{Y}$ axis to highlight low intensity peaks such as the $\mathrm{Cr}$ and $\mathrm{Pt}$. 